\title{
Safety of Endoscopic-Ultrasound-Guided Portal Injection Chemotherapy using Drug-Eluting Microbeads in a Porcine Model
}

\author{
Douglas O. Faigel ${ }^{1,{ }^{*}}$, Vijay P. Singh ${ }^{1}$, Krutika Patel ${ }^{1}$, Alaa El Chami ${ }^{1}$, Catherine C. \\ Raymond ${ }^{2}$, Tracy L. Landreth ${ }^{2}$, Ronald J. Marler ${ }^{3}$, Douglas F. Lake ${ }^{4}$ and Toufic Kachaamy ${ }^{5}$ \\ ${ }^{1}$ Division of Gastroenterology and Hepatology; ${ }^{2}$ Center for Procedural Innovation, and ${ }^{3}$ Research \\ Laboratories; ${ }^{4}$ Mayo Clinic, Scottsdale, Arizona; Arizona State University; ${ }^{5}$ Tempe, Arizona; and Cancer \\ Treatment Centers of America, Phoenix, Arizona, USA
}

\begin{abstract}
Background and Aims: Patients with diffuse liver metastases have systemic chemotherapy as their only treatment option. We developed Endoscopic Ultrasound (EUS)-guided portal injection chemotherapy (EPIC) to increase drug levels in hepatic tissue as a novel new liver directed therapy.

Methods: Sixteen anesthetized pigs were treated with $50 \mathrm{mg}$ of irinotecan $(n=8)$ or doxorubicin $(n=8)$. Half $(n=4)$ of the animals in each drug group were treated with EPIC-injected microbeads or EUS-guided chemotherapy without beads into the inferior vena cava (control). Animals were observed twice daily for 7 days for signs of clinical toxicities. Tissue samples were harvested for histology and drug levels. Blood counts and chemistries were determined pre-treatment and at 7 days.

Results: No toxicities as evidenced by abnormal animal behavior were observed. No significant changes occurred in blood chemistry or blood counts in the irinotecan groups. For doxorubicin, systemic injection significantly decreased albumin, hemoglobin, and white blood cell count $(P<.05)$, with no changes after EPIC. Hepatic histology showed mild foreign body reactions around the beads. No significant histologic changes were seen in other tissue sites. Neither irinotecan nor SN-38 was detectable at 7 days. For doxorubicin, no drug was detected in the plasma or bone marrow. The mean (SD) doxorubicin hepatic levels were non-significantly increased with EPIC vs control (181 [241] vs 151 [67] $\mathrm{ng} / \mathrm{g}$ ). Cardiac doxorubicin levels were significantly lower with EPIC (15 [4] vs 138 [48] ng/g; $P=.02$ ).

Conclusions: EPIC using drug-eluting microbeads was safe in this animal model. For doxorubicin, EPIC may be safer than systemic injection.
\end{abstract}

Keywords: Chemotherapy, doxorubicin, endoscopic ultrasound, irinotecan, safety.

\section{INTRODUCTION}

The liver is the most common site for metastases for a number of common malignancies. Patients with diffuse bilateral metastases are not candidates for liverdirected therapy and are treated with systemic chemotherapy. Systemic chemotherapy may be limited by inadequate hepatic tissue levels limiting efficacy or systemic toxicity. We developed endoscopic ultrasound-guided (EUS-guided) portal injection chemotherapy (EPIC) to overcome these limitations of systemic chemotherapy and as a novel liver-directed option for patients with diffuse bilateral metastases [1]. EPIC is performed using either drug-eluting microbeads [2-4] or albumin-bound nanoparticles [5] injected under EUS guidance transgastrically or transduodenally into the portal vein. The microbeads are carried downstream where they lodge in portal venules delivering the drug directly into the liver. The transgastric and transduodenal route has advantages over the transhepatic route in that it does not traverse potentially tumor-bearing liver tissue. We have reported

*Address correspondence to this author at the Division of Gastroenterology and Hepatology, Mayo Clinic, 13400 E Shea Blvd, Scottsdale, AZ 85259, USA; Tel: 480-301-6990; Fax: 480-301-6737; E-mail: faigel.douglas@mayo.edu that EPIC increases drug levels in hepatic tissue while sparing systemic drug exposure [1]. In 24 pigs treated with irinotecan, doxorubicin, or paclitaxel, we showed that at 1 hour EPIC resulted in increases in hepatic tissue levels and decreases in systemic levels of the drugs compared with systemic injections [1].

For the current study, we hypothesized that EPIC would have a similar or improved safety profile as a systemic venous injection of the same drug. Therefore, we sought to determine the safety of EPIC in a porcine survival model by comparing irinotecan or doxorubicinloaded microbeads with the same dose of unloaded drug injected into the inferior vena cava (IVC) under EUS guidance.

\section{METHODS}

This study was approved by the Mayo Clinic Institutional Animal Care and Use Committee on June 4, 2014.

The study was performed by using 16 male, $35-\mathrm{kg}$ anesthetized pigs. An experienced endoscopist (DOF) with more than 20 years' experience in EUS and EUS guided needle aspiration and therapeutics performed 
all procedures. A linear array EUS scope (GFUC140PAL5, Olympus America, Center Valley, Pennsylvania) was inserted perorally, and the portal vein or IVC was identified by anatomical landmarks and by pulse wave Doppler signature and punctured using standard, 22gauge, fine-needle aspiration needles (Boston Scientific, Marlborough, Massachusetts) via a transgastric route (direct puncture) as previously described [1]. Transhepatic puncture was not performed. For each drug, 8 animals were treated: 4 animals received EPIC treatment and 4 received a systemic (IVC) injection (control). No animal received more than 1 drug or more than 1 route. Irinotecan (50 $\mathrm{mg}$ ) or doxorubicin $(50 \mathrm{mg})$ was loaded onto $70-$ to 150-micron microbeads (LC Beads; Biocompatibles, London, UK) for EPIC. Control animals underwent an EUS-guided injection of the same dose of drug (not loaded onto beads) into the IVC. Signs of bleeding post-injection were assessed by EUS and color-flow Doppler such as active leakage or development of a perivascular collection or thrombus. EUS with Doppler was also used to assess vessel patency before, during and after the injection procedure.

The endpoints of the study were safety as defined by clinical observation, change in laboratory parameters and histological changes. Animals were observed twice daily for well-being, behavior, interactivity, and signs of toxicity (vomiting, diarrhea, anorexia, fever). On day 7 , the animals were euthanized, and tissue samples from the liver, skeletal muscle, bone marrow, and left ventricle (for doxorubicin) were obtained for histologic analyses and drug levels. In addition, full-thickness small intestine was obtained for histologic analysis. Whole blood and plasma were obtained at baseline and on day 7 for blood chemistry analysis and blood cell counts. Plasma and tissue levels of each drug and of the irinotecan metabolite $\mathrm{SN}-38$ were determined by liquid chromatography-mass spectroscopy. Tissue levels were measured after mechanical emulsification of specimens into a liquid and the results expressed as $\mathrm{ng} / \mathrm{g}$ of tissue. Histologic examination of formalin-fixed tissue samples after hematoxylin and eosin staining was performed by a veterinary pathologist blinded to treatment.

Whole blood was analyzed using manufacturer recommended methodology for electrolytes, $\mathrm{pH}$, total carbon dioxide, glucose, ionized calcium, hemoglobin (i-STAT Handheld analyzer, Abbott Point of Care Inc, Princeton, New Jersey) and reagent kits for bilirubin, alanine aminotransferase, alkaline phosphatase, albumin, and protein (Pointe Scientific Inc, Canton, Michigan). White blood cell counts were done manually using a hemocytometer with a Neubauer grid. The paired $t$ test was used to calculate $P$ values, and an $\alpha$ level of .05 was considered to be significant. Sample size was estimated at 4 animals per group, assuming a 4-fold change in hepatic drug concentrations, using a 1 -sided test (power $>80 \%, \alpha=.05$ ).

\section{RESULTS}

The EUS procedures were successful in all 16 animals. No immediate adverse effects were observed. No immediate damage to the blood vessels, leakage, bleeding, or effects on vessel patency were observed using EUS with Doppler. During the 7 days of observation, all animals in both groups were observed at least twice daily and all behaved appropriately, ate, and drank normally without signs of toxicities. No significant changes in blood cell counts or blood chemistry analyses were observed after delivery of irinotecan (Table 1). At necropsy no evident damage due to the EUS procedures (fluid collections, hematomas) were seen. In both groups of animals, protein levels increased. The albumin level decreased after EPIC with irinotecan, but not below the reference range. Systemic injection of doxorubicin resulted in significant decreases in albumin, hemoglobin, and white blood cell count $(P<.05)$ (Table 2). No significant changes were seen after EPIC with doxorubicin.

Plasma and tissue levels of each drug were determined at 7 days. Neither irinotecan nor the active metabolite SN-38 could be detected in either group in plasma, liver, bone marrow, or skeletal muscle. Doxorubicin could not be detected in the plasma or bone marrow. The mean (SD) levels of doxorubicin after EPIC vs controls were increased in the liver, but not significantly (181 [241] vs 151 [67] ng/g). Cardiac levels of doxorubicin were significantly less in the EPIC group (15 [4] vs 138 [48] ng/g; $P=.02$ ).

Histologic examinations showed mild foreign body reactions to the beads in the liver to the same degree in both the irinotecan and doxorubicin groups (Figure 1). No other significant histologic abnormalities or differences between groups were seen in the liver, heart, bone marrow, or intestine. No beads were seen in tissues outside of the liver.

\section{DISCUSSION}

Patients with diffuse metastases to the liver have limited treatment options. Liver-directed therapy may 
Table 1: Laboratory Results of Irinotecan Injection $(50 \mathrm{mg}$ )

\begin{tabular}{|c|c|c|c|c|}
\hline \multirow{2}{*}{ Result } & \multicolumn{2}{|c|}{ Control } & \multicolumn{2}{|c|}{ EPIC } \\
\hline & Day 0 & Day 7 & Day 0 & Day 7 \\
\hline $\mathrm{pH}$ & 7.46 & 7.46 & 7.41 & 7.44 \\
\hline Sodium, $\mathrm{mmol} / \mathrm{L}$ & 141 & 139 & 141 & 139 \\
\hline Potassium, mmol/L & 3.8 & 3.8 & 3.8 & 3.8 \\
\hline lonized calcium, mmol/L & 1.2 & 1.2 & 1.2 & 1.2 \\
\hline $\mathrm{TCO}_{2}, \mathrm{mmol} / \mathrm{L}$ & 29 & 34 & 30 & 31 \\
\hline Glucose, mg/dL & 63 & 69 & 98 & 92 \\
\hline Bilirubin, mg/dL & 0.25 & 0.18 & 0.15 & 0.15 \\
\hline $\mathrm{ALT}, \mathrm{IU} / \mathrm{L}$ & 22 & 24 & 25 & 21 \\
\hline Protein, g/dL & 4.4 & $5.1^{\mathrm{a}}$ & 4.5 & $5.2^{\mathrm{a}}$ \\
\hline Albumin, $\mathrm{g} / \mathrm{dL}$ & 4.2 & 4.3 & 5.2 & $4.2^{\mathrm{a}}$ \\
\hline Alkaline phosphatase, IU/L & 188 & 160 & 184 & 157 \\
\hline Hemoglobin, g/dL & 7.1 & 7.3 & 8.8 & 8 \\
\hline White blood cell count, $\mathrm{K} / \mathrm{mcL}$ & 16.5 & 15.8 & 18.1 & 18.2 \\
\hline
\end{tabular}

Abbreviations: ALT, alanine aminotransferase; EPIC, endoscopic ultrasound-guided portal injection chemotherapy; $\mathrm{TCO}_{2}$, total carbon dioxide.

${ }^{\mathrm{a} P} \mathrm{P}<.05$ vs Day 0.

Table 2: Laboratory Results of Doxorubicin Injection (50 mg)

\begin{tabular}{|c|c|c|c|c|}
\hline \multirow{2}{*}{ Result } & \multicolumn{2}{|c|}{ Control } & \multicolumn{2}{|c|}{ EPIC } \\
\hline & Day 0 & Day 7 & Day 0 & Day 7 \\
\hline $\mathrm{pH}$ & 7.4 & 7.4 & 7.4 & 7.4 \\
\hline Sodium, mmol/L & 141 & 139 & 140 & 139 \\
\hline Potassium, $\mathrm{mmol} / \mathrm{L}$ & 4.1 & 3.6 & 4.1 & 3.7 \\
\hline Ionized calcium, $\mathrm{mmol} / \mathrm{L}$ & 1.1 & 1.2 & 1.1 & 1.2 \\
\hline $\mathrm{TCO}_{2}, \mathrm{mmol} / \mathrm{L}$ & 31 & 31 & 30 & 28 \\
\hline Glucose, mg/dL & 83 & 89 & 91 & 83 \\
\hline Bilirubin, mg/dL & 0.5 & 0.1 & 0.4 & 0.2 \\
\hline ALT, IU/L & 22.5 & 23.1 & 25.8 & 25.2 \\
\hline Protein, g/dL & 4.6 & 4 & 4.7 & 5.1 \\
\hline Albumin, $\mathrm{g} / \mathrm{dL}$ & 4.2 & $3.7^{\mathrm{a}}$ & 4.1 & 4.1 \\
\hline Alkaline phosphatase, IU/L & 179 & 124 & 205 & 131 \\
\hline Hemoglobin, g/dL & 8.7 & $7.1^{\mathrm{a}}$ & 8.9 & 7.9 \\
\hline White blood cell count, $\mathrm{K} / \mathrm{mcL}$ & 22.6 & $12^{a}$ & 21.6 & 21.3 \\
\hline
\end{tabular}

Abbreviations: ALT, alanine aminotransferase; EPIC, endoscopic ultrasound-guided portal injection chemotherapy; $\mathrm{TcO}_{2}$, total carbon dioxide. ${ }^{\mathrm{a}} P<.05$ vs Day 0.

be appropriate for focal metastases and patients with oligometatstatic disease. These options include chemoembolization, image modulated radiotherapy, radiofrequency ablation and resection [6-8]. Unfortunately, patients with diffuse disease involving both lobes of the liver, particularly when the lesions are small and numerous, do not have these options and are therefore treated with systemic chemotherapy $[9,10]$. Dose-limiting toxicities may negatively impact both patients' quality of life and the effectiveness of systemic chemotherapy, and as the bile ducts derive all of their oxygen from the hepatic arteries bilateral 


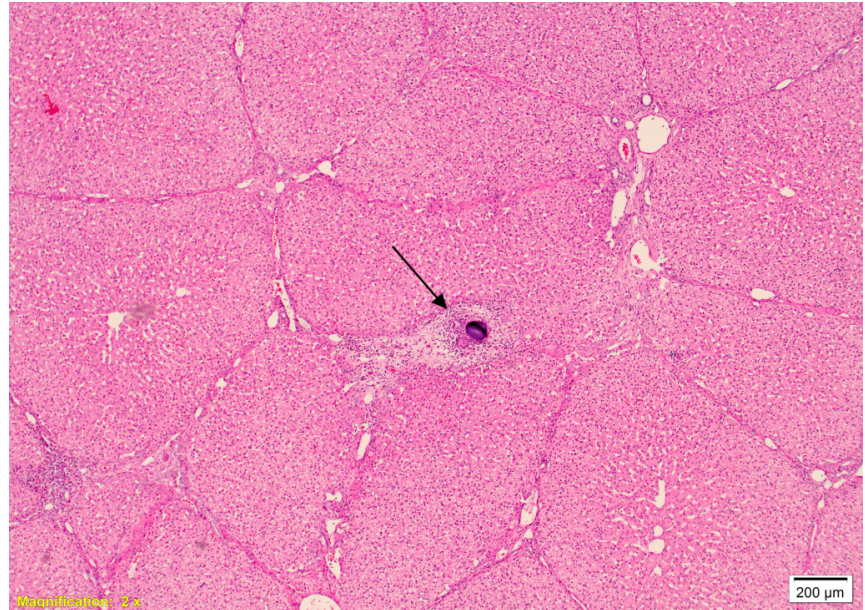

A

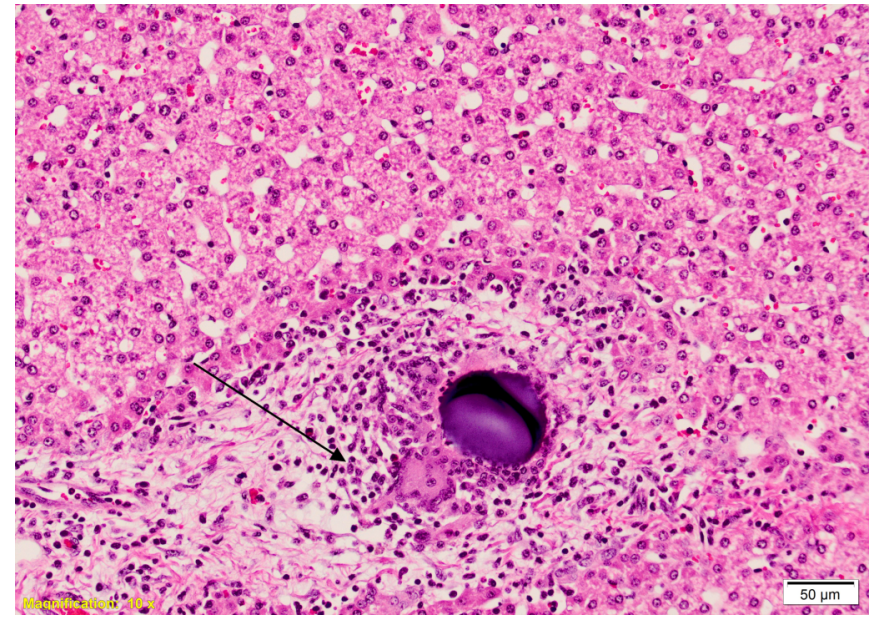

B

Figure 1: Photomicrographs of microbeads in the liver after endoscopic ultrasound-guided portal injection chemotherapy with doxorubicin. Foreign body reactions (arrow) can be seen around the microbeads. A, hematoxylin-eosin, original magnification $\times 2$. B, hematoxylin-eosin, original magnification $\times 10$.

arterial chemoembolization is not performed [11,12]. We developed EPIC to overcome these limitations to provide a new liver-directed treatment for patients in whom systemic chemotherapy is their only option. We previously demonstrated the feasibility of this approach in a porcine nonsurvival model [1].

In the current study, we examined the safety and toxicity of EPIC using irinotecan- or doxorubicin-loaded microbeads in a 7-day porcine survival model. We compared drug delivery by the EPIC method with delivery of the same drug, not loaded onto beads, injected into the systemic venous system (IVC). In our prior study, we chose a $100-\mathrm{mg}$ dose to approximate the typical, intravenous systemic dose used for cancer patients. We found that EPIC caused a doubling of hepatic irinotecan levels and a 5-fold increase in hepatic doxorubicin levels at one hour [1]. In designing the current survival study, we took into account that higher hepatic levels could result in toxicity and chose doses to approximate a starting dose in a phase 1 , dose-escalation trial. Therefore, for this safety study, we used 50-mg doses because we felt that this dose would achieve hepatic levels at least as high as the $100-\mathrm{mg}$ systemic dose. To control for the effects of EUS-guided chemotherapy injections, we also used EUS guidance to inject unloaded drug into the IVC of the control animals; thus, both the EPIC and control groups received chemotherapy by the EUS-guided approach (EPIC with microbeads, IVC without microbeads).

The EUS procedures were performed in all 16 animals without evident adverse events or toxicities.
During the 7 days of observation, all animals behaved appropriately, ate, and drank normally. Histologic examination of the liver tissue showed mild foreign body reactions around the beads, with both irinotecan and doxorubicin loading. Although these histologic changes are of doubtful importance, it should be noted that the beads are not biodegradable $[13,14]$. Laboratory evaluations at baseline and at 7 days did not show significant changes in the irinotecan control groups. In the EPIC group, albumin decreased from 5.2 to $4.2 \mathrm{~g} / \mathrm{dL}(P<.05)$; however, the post-EPIC level is similar to that of the baseline level in the other 3 groups and thus not likely to represent an important observation. For doxorubicin, systemic injection but not EPIC was associated with myelosuppression, as evidenced by significant decreases in hemoglobin level and white blood cell count.

Differences existed in irinotecan and doxorubicin levels at 7 days. Irinotecan and the active metabolite SN-38 could not be detected in plasma or tissue at 7 days. Irinotecan is known to bind less well to beads than doxorubicin $[13,14]$. In our prior study, the 1-hour levels of irinotecan were double in the liver and half that of the control group in the plasma and systemic tissues [1]. How long this differential persists and the potential clinical implications remain unclear. In the current study, doxorubicin persisted in liver and heart tissue at 7 days in both groups. The levels in the liver were $20 \%$ higher with EPIC, but this was not statistically significant. The cardiac levels of doxorubicin were 9-fold higher in the control animals. This is a potentially clinically significant finding because doxorubicin is cardiotoxic [15]. In our prior study, 
cardiac levels at 1 hour were 30 -fold higher in the control animals [1]. Thus, the difference in cardiac levels is substantial, occurs immediately, and persists for at least 1 week.

This study has several limitations. A healthy animal model was used because an appropriate animal model for metastases does not exist, and safety beyond 1 week was not determined. We also did not evaluate higher doses of drug. Nor did we evaluate multiple injections, which may be important because the microbeads are not biodegradable $[13,14]$.

Whether human cancer patients would tolerate this treatment or derive clinical benefit can only be determined in appropriately designed trials. The appropriate comparator to EPIC would be systemic chemotherapy as the target population would be patients with diffuse bilateral hepatic metastases in whom other liver directed therapies would not be indicated. In the US these patients are not candidates for chemoembolization, radiotherapy, radiofrequency ablation or resection, and hepatic arterial infusion is rarely performed. We hypothesize that EPIC using drug-eluting microbeads may be effective through three possible mechanisms. The first, and likely most important is increased drug delivery to the hepatic tumors themselves as opposed to systemic chemotherapy. Second is the prolonged drug levels likely achievable using drug eluting beads as opposed to systemic injection chemotherapy. Third is the potential effect on the circulating population of cancer cells.

The natural history of liver metastases begins with attachment to the hepatic sinusoids where the cancer cells interact with sinusoidal endothelial cells, stellate cells and Kupfer cells [16]. The blood flow in the hepatic sinusoids is mostly derived from the portal vein. As the cancer cells grow and invade and the tumor becomes larger hypoxia triggers neovascularization from the hepatic arteries [16,17]. Thus, the oxygen supply to the tumor is similar to the liver in that it is dual with smaller tumors likely deriving greater percentages of oxygen from the portal vein and larger tumors from the hepatic artery after neovascularization has occurred. This dual nature is evident in recent studies of hepatic artery chemotherapy infusion where the efficacy is enhanced by clamping the PV thus forcing all of the blood supply to be from the HA negating a dilutional effect from the PV $[18,19]$. As the chemotherapeutic agents elute off of the beads the drug may reach the tumor by direct diffusion through adjacent tissue, blood flow through portal venules to the peritumoral sinusoids, portal venous inflow into the tumors, and systemically as the drug circulates back to the tumor through the systemic circulation.

A second important potential mechanism of action of EPIC with microbeads is the prolonged presence of drug in tissues and circulation as opposed to single episodic systemic injections of chemotherapy. Intraarterial injection of irinotecan and doxorubicin loaded microbeads results in high hepatic levels, low systemic levels and drug that persists up to three months $[13,14]$. In this 7-day study doxorubicin loaded microbeads resulted in similar hepatic levels as systemic injection of unloaded drug (181 (241) vs 151 (67) ng/gm, EPIC vs Control), but cardiac doxorubicin levels were significantly lower with EPIC (15 (4) vs. 138 (48) ng/gm, $p=0.015)$, an important achievement given the cardiotoxic effects of this drug. We also observed less doxorubicin-induced myelosuppression with EPIC. This highlights the possibility that even if enhanced efficacy is not achieved, similar efficacy to systemic chemotherapy with less toxicity may be achievable.

A third hypothesis as to how EPIC may be effective is the effect on circulating cancer cells. It is now known that most if not all epithelial cancers such as pancreas cancer circulate in the blood stream [20]. Thus, these cancer cells will pass through the liver multiple times per day. With EPIC, the microbeads create zones of high drug concentration through which these circulating cancer cells must pass. It is possible that these multiple exposures as the cancer cells pass in proximity to the drug eluting microbeads will have a therapeutic benefit. This hypothesis has yet to be specifically tested.

In conclusion, using EPIC with drug-eluting microbeads appears to be safe and well tolerated in the porcine survival model. For irinotecan, EPIC is at least as safe as a systemic injection. For doxorubicin, EPIC may be safer: we found less myelosuppression and lower levels of drug in cardiac tissue persisting for at least 7 days. Clinical benefits in human cancer patients who are not candidates for other liver-directed therapies should be tested.

\section{ACKNOWLEDGEMENTS}

This study was supported by a Cancer Treatment Centers of America grant. The EUS aspiration needles were donated by Boston Scientific. 


\section{AUTHOR CONTRIBUTIONS}

Faigel: Conception and design; analysis and interpretation of the data; drafting of the article; critical revision of the article for important intellectual content; final approval of the article.

Kachaamy: Conception and design; analysis and interpretation of the data; final approval of the article.

Lake: Conception and design; analysis and interpretation of the data; critical revision of the article for important intellectual content; final approval of the article.

Singh: Analysis and interpretation of the data; drafting of the article; critical revision of the article for important intellectual content; final approval of the article.

Patel: Analysis and interpretation of the data; final approval of the article

El Chami: Conception and design; analysis and interpretation of the data; final approval of the article.

Raymond: Conception and design; critical revision of the article for important intellectual content; final approval of the article.

Landreth: Conception and design; critical revision of the article for important intellectual content; final approval of the article.

Marler: Conception and design; analysis and interpretation of the data; drafting of the article; critical revision of the article for important intellectual content; final approval of the article.

\section{CONFLICT OF INTEREST}

None.

\section{ABBREVIATIONS}

$$
\begin{aligned}
& \text { EPIC }=\text { EUS-guided portal injection chemotherapy } \\
& \text { EUS }=\text { endoscopic ultrasound } \\
& \text { IVC }=\text { inferior vena cava }
\end{aligned}
$$

\section{REFERENCES}

[1] Faigel DO, Lake DF, Landreth TL, Kelman CC, Marler RJ. EUS-guided portal injection chemotherapy for treatment of hepatic metastases: feasibility in the acute porcine model. Gastrointest Endosc 2016; 83(2): 444-6. https://doi.org/10.1016/j.gie.2015.08.064
[2]

Martin RC, Robbins K, Tomalty D, et al. Transarterial chemoembolisation (TACE) using irinotecan-loaded beads for the treatment of unresectable metastases to the liver in patients with colorectal cancer: an interim report. World $J$ Surg Oncol 2009; 7: 80. https://doi.org/10.1186/1477-7819-7-80

[3] Martin R, Geller D, Espat J, et al. Safety and efficacy of trans arterial chemoembolization with drug-eluting beads in hepatocellular cancer: a systematic review. Hepatogastroenterology 2012; 59(113): 255-60.

[4] Martin RC, Joshi J, Robbins K, et al. Hepatic intra-arterial injection of drug-eluting bead, irinotecan (DEBIRI) in unresectable colorectal liver metastases refractory to systemic chemotherapy: results of multi-institutional study. Ann Surg Oncol 2011; 18(1): 192-8. https://doi.org/10.1245/s10434-010-1288-5

[5] Viudez A, Ramirez N, Hernandez-Garcia I, Carvalho FL, Vera R, Hidalgo M. Nab-paclitaxel: a flattering facelift. Crit Rev Oncol Hematol 2014; 92(3): 166-80. https://doi.org/10.1016/j.critrevonc.2014.06.001

[6] Clark ME, Smith RR. Liver-directed therapies in metastatic colorectal cancer. J Gastrointest Oncol 2014; 5(5): 374-87.

[7] Xing M, Kooby DA, El-Rayes BF, Kokabi N, Camacho JC, Kim HS. Locoregional therapies for metastatic colorectal carcinoma to the liver: an evidence-based review. J Surg Oncol 2014; 110(2): 182-96. https://doi.org/10.1002/jso.23619

[8] Nguyen KT, Gamblin TC, Geller DA. World review of laparoscopic liver resection: 2,804 patients. Ann Surg 2009; 250(5): 831-41. https://doi.org/10.1097/SLA.0b013e3181b0c4df

[9] Strickler JH, Hurwitz HI. Palliative treatment of metastatic colorectal cancer: what is the optimal approach? Curr Oncol Rep 2014; 16(1): 363. https://doi.org/10.1007/s11912-013-0363-z

[10] Michl P, Gress TM. Current concepts and novel targets in advanced pancreatic cancer. Gut 2013; 62(2): 317-26. https://doi.org/10.1136/gutjnl-2012-303588

[11] Saltz L. Systemic therapy for metastatic colorectal cancer. $J$ Natl Compr Canc Netw 2013; 11(5 Suppl): 649-52. https://doi.org/10.6004/jnccn.2013.0193

[12] Thota R, Pauff JM, Berlin JD. Treatment of metastatic pancreatic adenocarcinoma: a review. Oncology (Williston Park) 2014; 28(1): 70-4.

[13] Taylor RR, Tang Y, Gonzalez MV, Stratford PW, Lewis AL. Irinotecan drug eluting beads for use in chemoembolization: in vitro and in vivo evaluation of drug release properties. Eur J Pharm Sci 2007; 30(1): 7-14. https://doi.org/10.1016/j.ejps.2006.09.002

[14] Namur J, Wassef M, Millot JM, Lewis AL, Manfait M, Laurent A. Drug-eluting beads for liver embolization: concentration of doxorubicin in tissue and in beads in a pig model. $J$ Vasc Interv Radiol 2010; 21(2): 259-67. Erratum in: J Vasc Interv Radiol 2010; 21(4): 596. https://doi.org/10.1016/j.jvir.2009.10.026

[15] Lotrionte M, Biondi-Zoccai G, Abbate A, et al. Review and meta-analysis of incidence and clinical predictors of anthracycline cardiotoxicity. Am J Cardiol 2013; 112(12): 1980-4.

https://doi.org/10.1016/..amjcard.2013.08.026

[16] Paschos KA, Majeed AW, Bird NC. Natural history of hepatic metastases from colorectal cancer - pathobiological pathways with clinical significance. World J Gastroenterol 2014; 20(14): 3719-3737.

https://doi.org/10.3748/wjg.v20.i14.3719

[17] Fukuda R, Kelly B, Semenza GL. Vascular Endothelial Growth Factor Gene Expression in Colon Cancer Cells Exposed to Prostaglandin $E_{2}$ Is Mediated by Hypoxiainducible Factor 1. Cancer Research 2003; 63: 2330-2334. 
[18] Ben-Shabat I, Hansson C, Sternby Eilard M, et al. Isolated hepatic perfusion as a treatment for liver metastases of uveal melanoma. J Vis Exp 2015; 95: e53490. https://doi.org/10.3791/52490

[19] Sperling J, Ziemann C, Gittler A, Benz-Weiser A, Menger $\mathrm{MD}$, Kollmar $\mathrm{O}$. Tumour growth of rat liver metastases is inhibited by hepatic arterial infusion of the mTOR-inhibitor temsirolimus after portal branch ligation. Clin Exp Metastasis 2015; 32(4): 313-21.

https://doi.org/10.1007/s10585-015-9707-4

[20] Xu Y, Li Jing, Wang $X$, et al. Detection of circulating tumor cells using negative enrichment immunofluorescence an in situ hybridization system in pancreatic cancer. Int J Mol Sci 2017; 18(4): 622.

https://doi.org/10.3390/ijms18040622

DOI: http://dx.doi.org/10.30683/1929-2279.2018.07.04.1

(C) 2018 Faigel et al.; Licensee Neoplasia Research.

This is an open access article licensed under the terms of the Creative Commons Attribution Non-Commercial License (http://creativecommons.org/licenses/by-nc/3.0/) which permits unrestricted, non-commercial use, distribution and reproduction in any medium, provided the work is properly cited. 